\title{
Multi-tyrosine kinase inhibitors in preclinical studies for pediatric CNS AT/RT: Evidence for synergy with Topoisomerase-I inhibition
}

\author{
Aarthi Jayanthan ${ }^{1}$, Delphine Bernoux ${ }^{1}$, Pinaki Bose ${ }^{2}$, Karl Riabowol $^{2}$ and Aru Narendran ${ }^{1 *}$
}

\begin{abstract}
Background: Currently, Atypical Teratoid Rhabdoid Tumor (AT/RT) constitutes one of the most difficult to treat malignancies in pediatrics. Hence, new knowledge of potential targets for therapeutics and the development of novel treatment approaches are urgently needed. We have evaluated the presence of cytokine pathways and the effects of two clinically available multi-tyrosine kinase inhibitors for cytotoxicity, target modulation and drug combinability against AT/RT cell lines.

Results: AT/RT cell lines expressed measurable quantities of VEGF, FGF, PDGF and SDF-1, although the absolute amounts varied between the cell lines. The targeted receptor tyrosine kinase inhibitor sorafenib inhibited the key signaling molecule Erk, which was activated following the addition of own conditioned media, suggesting the existence of autocrine/paracrine growth stimulatory pathways. The multi-tyrosine kinase inhibitors sorafenib and sunitinib also showed significant growth inhibition of AT/RT cells and their activity was enhanced by combination with the topoisomerase inhibitor, irinotecan. The loss of cytoplasmic NF-kappa-B in response to irinotecan was diminished by sorafenib, providing evidence for a possible benefit for this drug combination.

Conclusions: In addition to previously described involvement of insulin like growth factor (IGF) family of cytokines, a multitude of other growth factors may contribute to the growth and survival of AT/RT cells. However, consistent with the heterogeneous nature of this tumor, quantitative and qualitative differences may exist among different tumor samples. Multi-tyrosine kinase inhibitors appear to have effective antitumor activity against all cell lines studied. In addition, the target modulation studies and drug combinability data provide the groundwork for additional studies and support the evaluation of these agents in future treatment protocols.
\end{abstract}

\section{Background}

Atypical Teratoid Rhabdoid Tumor of the central nervous system (CNS AT/RT) is a highly malignant neoplasm of infants and young children. A biallelic inactivation of the hSnf5/Ini1 gene located in 22q11.2 is a characteristic molecular defect in these tumors [1-4]. Murine knock-out models have confirmed that hSnf5/Ini1 is a tumor-suppressor gene [5], but the details of its exact role in the initiation and growth of the AT/RT are still being investigated. To date, studies showing INI1 interaction with key signaling

\footnotetext{
* Correspondence: anarendr@ucalgary.ca

'Laboratory for Pre-clinical and Drug Discovery Studies, Pediatric Oncology Experimental Therapeutics Investigators Consortium (POETIC) and Division of Pediatric Oncology, Alberta Children's Hospital, 2888 Shaganappi Trail NW,

Calgary, T3B 6A8, Canada

Full list of author information is available at the end of the article
}

molecules suggest its potential to modify the response to factors that mediate cell growth and differentiation programs [6-8]. There is emerging evidence for the existence of autocrine and/or paracrine growth factor signaling pathways in these cells. Previously, we were able to maintain disseminated AT/RT cells in culture by the addition of autologous CSF to culture medium [9]. Agents that inhibit IGF-IR activity have been shown to diminish tumor cell growth and targeting of IGF-IR expression with antisense oligonucleotides resulted in increased apoptosis and sensitivity to a number of chemotherapeutic agents [10]. Furthermore, Arcaro and colleagues have shown evidence for autocrine signaling by insulin and its receptor (IR) in AT/RT cells, which involves the PI3K/Akt pathway [11]. These findings suggest that abnormally regulated cytokine

\section{Ciomed Central}


pathways and their downstream signaling molecules can be effective targets for therapeutics in AT/RT.

Ultra structurally, AT/RT often presents as a polymorphous tumor with overlapping morphologic features consisting of primitive neuroectodermal tumor (PNET), mesenchymal, rhabdoid and epithelial components. This phenotypic heterogeneity is likely to be aided by multilevel cross stimulation of growth and survival pathways and signaling molecules. As such, a single-targeted agent may not be the optimal choice, as these agents may permit the development of salvage or escape mechanisms. However, by virtue of their ability to interfere with a diverse array of signaling molecules, including cytokine receptor kinases, multi-targeted inhibitors may provide a therapeutic advantage in the treatment of AT/RT. In the recent past, tyrosine kinase inhibitors with multiple targets have been found to have clinically achievable activity and acceptable tolerability in studies against heterogeneous malignancies [12]. In this study, we have evaluated two such agents, sunitinib and sorafenib, for in vitro activity and drug combinability against three AT/RT cell lines.

\section{Results}

\section{Cytokine expression by AT/RT cells}

Quantitative evaluation of the cytokines found in the culture supernatants of the three AT/RT cell lines was performed by multiplex assay. Data presented in Table 1 shows significant presence of six of the 65 cytokines examined. However, with the exception of VEGF, other cytokines showed quantitative variations between the cell lines. For example, in comparison to BT12 and KCCF1 cells, BT16 cells did not express measurable levels of IL-8 and MCP-1, and expressed only a very low level of SDF-1. Although BT12 supernatants contained higher quantities of all other cytokines, the level of FGF(b) was measurably lower in this sample, indicating the potential heterogeneity in the presence of different cytokines in the tumor microenvironment.

\section{Sensitivity of AT/RT cell lines to multi-targeted tyrosine kinase inhibitors and irinotecan}

The presence of a multitude of cytokines in the culture supernatants of the AT/RT cell lines indicated the potential for autocrine or paracrine growth sustaining processes

Table 1 Quantitative analysis of cytokines present in the spent culture supernatants of AT/RT cell lines

\begin{tabular}{lcccccc}
\hline Cytokine $(\boldsymbol{\rho g} / \mathbf{m l})$ & FGF(b) & IL-8 & MCP-1 & PDGF-AA & VEGF & SDF-1 \\
\hline Media Control & UD & UD & UD & UD & 16.26 & UD \\
\hline BT 12 Supernatant & 14.93 & 144.7 & 63.38 & 157.76 & 226.67 & 228.28 \\
\hline BT 16 Supernatant & 82.45 & UD & UD & 197.06 & 261.04 & 4.74 \\
\hline KCCF1 Supernatant & 40.35 & 10.18 & UD & 43.35 & 124.57 & 190.17 \\
\hline UD - undetectable & & & & & &
\end{tabular}

utilizing these molecules. Therefore, we wanted to investigate the effects of agents that have been shown to interfere with the activity of such receptor pathways. Sorafenib and sunitinib have been shown to inhibit the activity of a number of cytokine receptors, including vascular endothelial growth factor receptor (VEGFR), platelet-derived growth factor receptor (PDGFR), stem cell factor receptor (c-Kit) and FMS-like tyrosine kinase-3 (Flt-3) [13,14]. In the next set of experiments, the three AT/RT cell lines were evaluated for sensitivity to sorafenib and sunitinib by in vitro cytotoxicity assays. Figures $1 \mathrm{~A}$ and $1 \mathrm{~B}$ show the dose dependent inhibition of AT/RT cell growth by these agents. From these data, $\mathrm{IC}_{50}$ values were calculated and presented in Table 2. $\mathrm{IC}_{50}$ values for each cell line ranged from 2.8 to $3.6 \mu \mathrm{M}$ for sorafenib and 3.2 to $3.7 \mu \mathrm{M}$ for sunitinib. As a means to further support the targeted inhibition of receptor pathways by sorafenib and sunitinib, the expression of proteins targeted by these inhibitors was determined by Western blot analysis. It was found that all three AT/RT cell lines expressed receptor tyrosine kinases c-Kit, PDGF-R $\beta$, VEGFR2 and Flt-3, as well as the intracellular targets of sorafenib, c-Raf and p38 $\alpha$ (Figure 2).

\section{Synergistic activity of irinotecan with sorafenib and sunitinib}

Previous studies have indicated the potential activity of the new generation topoisomerase I inhibitor irinotecan against brain tumors and its ability to increase the activity of agents that block VEGF activity [15]. To deduce the role of irinotecan in potential combination therapies, we first analyzed its activity as a single agent. Figure 3 shows the cytotoxic effects of irinotecan against the three AT/RT cell lines. The $\mathrm{IC}_{50}$ values (Table 2 ) ranged from 2.0 to $6.7 \mu \mathrm{M}$, with BT12 cell line exhibiting a significantly lower IC50 value of $2 \mu \mathrm{M}$ compared to other two cell lines. We also tested the activity of the active metabolite of irinotecan, SN-38 against the three AT/RT cell lines. The $\mathrm{IC}_{50}$ values (Table 2) ranged from 0.03 to $4.6 \mu \mathrm{M}$, with KCCF1 exhibiting a significantly higher $\mathrm{IC}_{50}$ value compared to the other two cell lines. Next, we evaluated the drug combinability of irinotecan with sorafenib and sunitinib. The ability of a fixed concentration of irinotecan $\left(\mathrm{IC}_{25}\right)$ to reduce the $\mathrm{IC}_{50}$ values in serially diluted sorafenib and sunitinib was evaluated by in vitro cytotoxicity assays and combination indices $(\mathrm{CI})$ were calculated according to the method of Chou and Talalay [16]. Table 3 shows the respective $\mathrm{CI}$ calculations. Combination index values less than $1(\mathrm{CI}<1)$ indicates synergy between two agents.

\section{Modulation of intracellular signaling molecules by sorafenib}

Our initial set of experiments involved the screening of changes in the activation status of signaling molecules in response to treatment with sorafenib in AT/RT cells. 

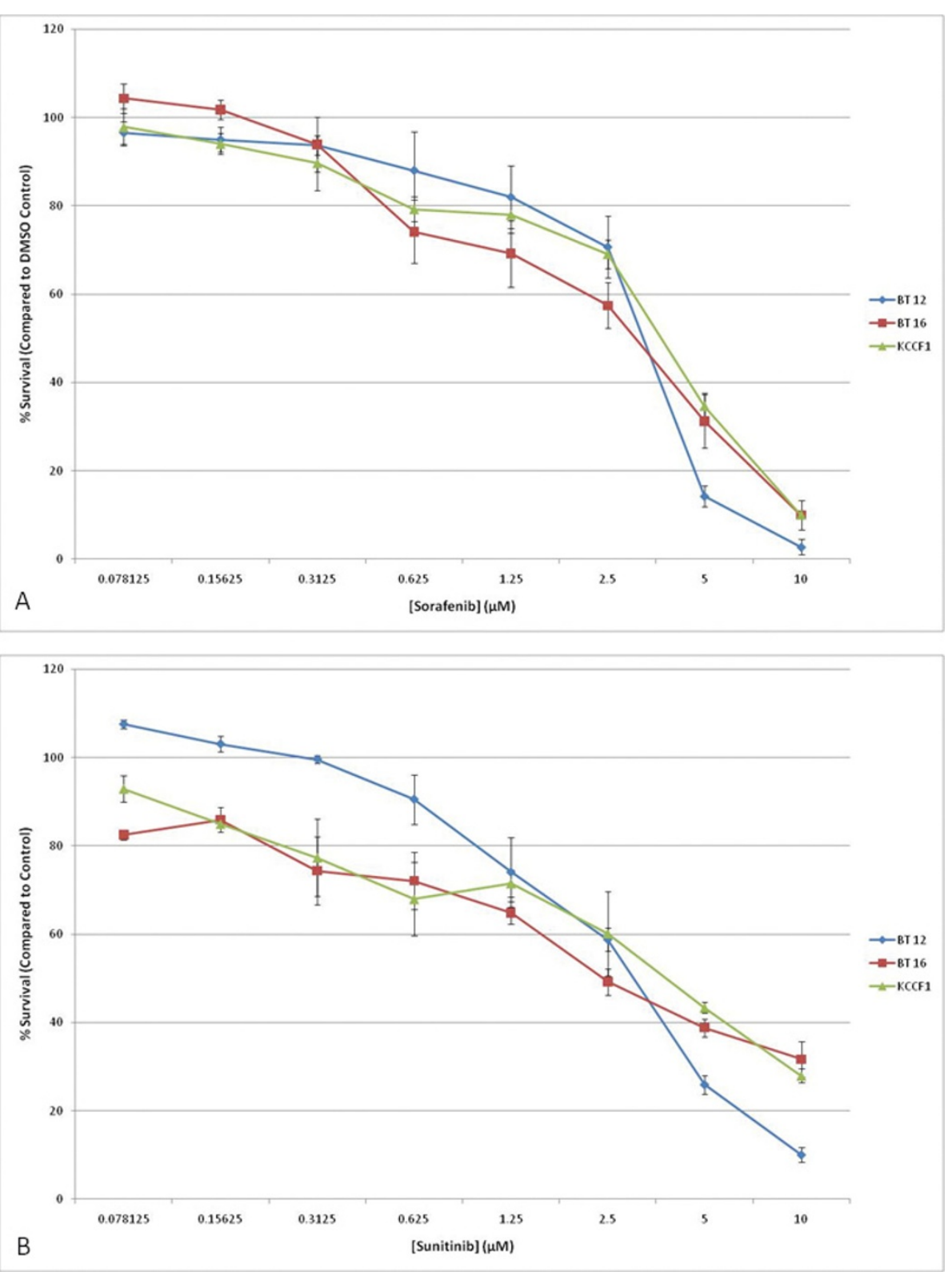

Figure 1 Sorafenib and sunitinib induce cell growth inhibition in AT/RT cell lines in vitro. Triplicate cultures of three AT/RT cell lines were incubated with increasing concentrations of sorafenib (A) or sunitinib (B). Appropriate dilutions of DMSO were also included as vehicle control. After four days in culture, cell viability was measured and plotted as a percentage of cell survival compared to equivalent vehicle control. Bars indicate SD. Data presented above are representative of five separate experiments. $\mathbf{C}_{50}$ values calculated from these data are given in Table 2.

Table 2 In vitro cytotoxicity of sorafenib, sunitinib, irinotecan and SN-38 against three AT/RT cell lines

\begin{tabular}{cccc}
\hline Inhibitor, IC $_{\mathbf{5 0}}(\boldsymbol{\mu M})$ & BT 12 & BT 16 & KCCF1 \\
\hline Sorafenib & 3.3 & 2.8 & 3.6 \\
\hline Sunitinib & 3.2 & 3.2 & 3.7 \\
\hline Irinotecan & 2.0 & 4.1 & 6.7 \\
\hline SN-38 & 0.09 & 0.03 & 4.6 \\
\hline
\end{tabular}

$\mathrm{IC}_{50}$ - half maximal inhibitory concentration
Exponentially growing cells were treated with $10 \mu \mathrm{M}$ of sorafenib, or appropriate vehicle control, and cell lysates were analyzed by Western blots as described in materials and methods. Data presented in Figure 4A shows that, in most cases, sorafenib decreased the levels of multiple signaling components in AT/RT cells Significant loss of phosphorylated cell growth regulators was seen in all AT/RT cells although variations were seen among the different cell lines; Erk1/2 (BT12, KCCF1), 


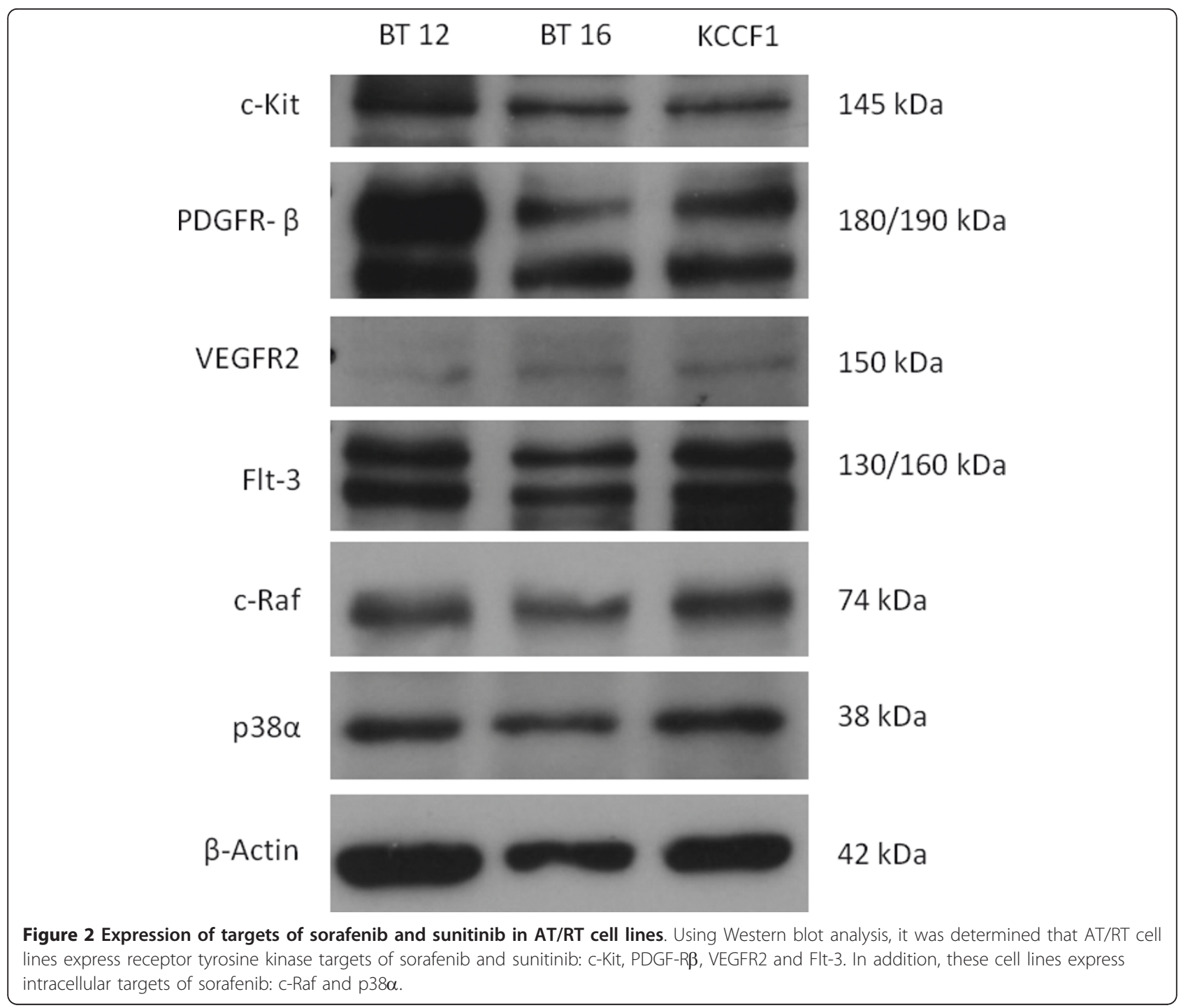

Akt 1/2 (BT12), c-Raf (BT12, BT16) and Stat3 (BT12, KCCF1). Loss of the cell survival molecule Mcl-1, however, was found in all three cell lines studied.

The addition of conditioned medium to cells that are serum starved provides an experimental model to study the autocrine/paracrine pathways mediated by secreted cytokines. Agents that block such activation pathways may contribute to ultimate growth inhibitory activities and provide a rationale for investigating receptor tyrosine kinase inhibitors as targeted therapeutics. In the next set of experiment we demonstrate that indeed the conditioned media from AT/RT cells induce Erk phosphorylation, which has been shown to be one of the downstream targets of sorafenib activity (Figure 4B).

Previous studies have suggested that the activation of NF-kappa-B (NF- $\kappa \mathrm{B})$ in response to chemotherapeutic agents, including irinotecan may relate to the generation of resistance in cancer cells $[17,18]$. To further evaluate the input of MTK inhibition in this process, we evaluated the effect on NF- $\kappa \mathrm{B}$ in response to irinotecan as a single agent and then in combination with sorafenib. Using BT12 cells, we examined the presence of cytoplasmic $\mathrm{NF}-\kappa \mathrm{B}$ by indirect immunofluorescence. Cells receiving sorafenib, irinotecan or the combination were fixed and stained with antibodies to NF- $\kappa$ B. The slides were visualized under a fluorescent microscope and random fields were photographed. Representative photographs in Figure $5 \mathrm{~A}$ show that the cytoplasmic staining of NF- $\kappa \mathrm{B}$ is unchanged in sorafenib treated cells compared to control cells, but there is a significant reduction in such staining when the cells were treated with irinotecan. However, this loss is reduced by combination with sorafenib. As activated $\mathrm{NK}-\kappa \mathrm{B}$ translocates from cytoplasm during the activation process, this indicates that irinotecan and sorafenib combination leads to potentially reduced translocation of NF- $\kappa \mathrm{B}$ compared to irinotecan alone. To further 


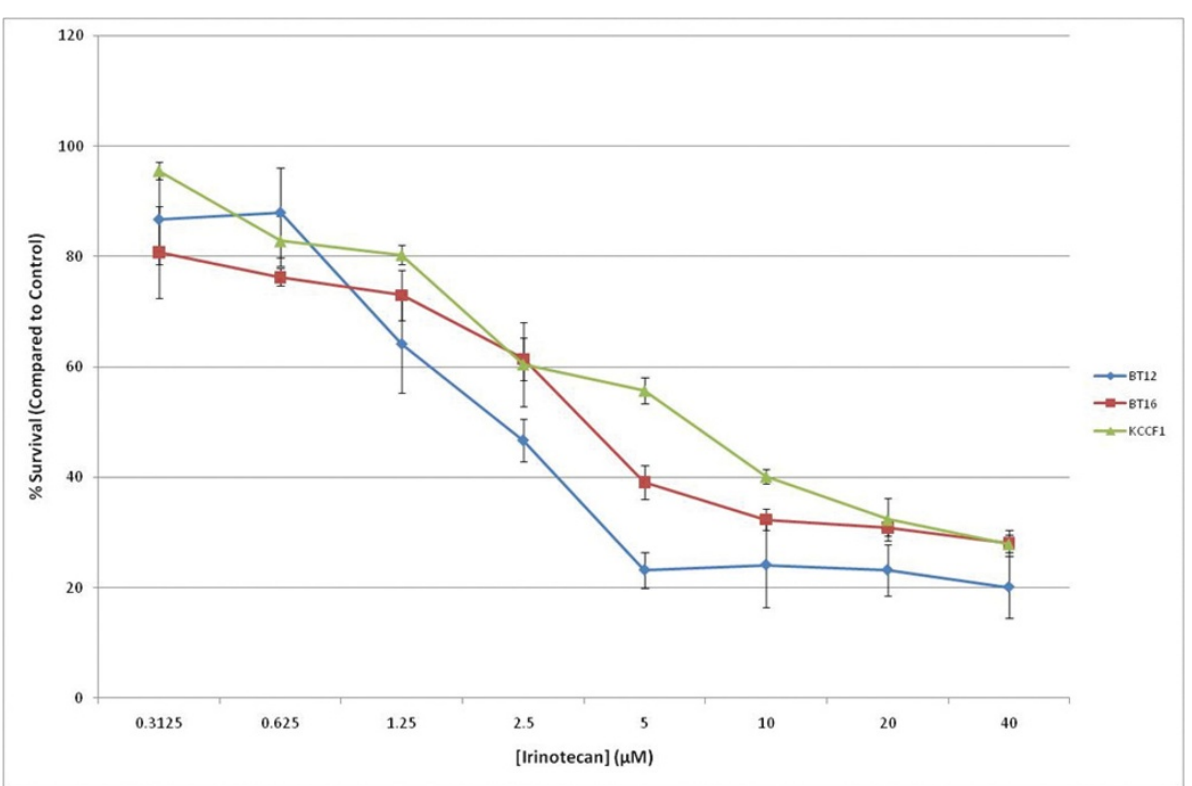

Figure 3 Irinotecan inhibits the in vitro growth of AT/RT cells. Triplicate cultures of AT/RT cell lines were cultured in the presence of increasing concentrations of irinotecan. Following four days in culture, cell growth inhibition was measured by Alamar blue assay. Percentage of cell survival was calculated by comparison of treated cells to control cells. Similar results were obtained in five separate experiments. $\boldsymbol{I}_{50}$ values obtained from these data are given in Table 2.

confirm this possibility, cytoplasmic extracts of cells treated with either irinotecan alone or the irinotecan and sorafenib combination were evaluated by Western blot analysis. Results presented in Figure $5 B$ show that NF- $\kappa$ B p65 detected with irinotecan alone is significantly less compared to levels detected when the cells received added sorafenib. This suggests that sorafenib may be able to reduce the translocation and hence the activation on $\mathrm{NF}-\kappa \mathrm{B}$ that follows irinotecan treatment. In addition, compared to treatment with sorafenib or irinotecan alone, the cells treated with the combination showed increased $\mathrm{I} \kappa \mathrm{B} \alpha$, providing further evidence for stabilization of NF- $\kappa \mathrm{B}$ under this condition.

Previous studies have shown that the tumor suppressor gene CDKN1B encodes for a 27-kDa cyclin-dependent kinase inhibitory protein, p27Kip1, which inhibits cell proliferation and motility [19]. Our initial screening studies (data not shown) have shown that AT/RT cells also down regulate p27Kip1 in response to irinotecan. Sorafenib, however, did not have this effect and the

Table 3 Combination indices in drug combination studies of irinotecan and the multi-kinase inhibitors sorafenib and sunitinib

\begin{tabular}{cccc}
\hline Inhibitor, Cl & BT 12 & BT 16 & KCCF1 \\
\hline Sorafenib & 0.7 & 0.8 & 0.9 \\
\hline Sunitinib & 0.9 & 0.3 & 0.4 \\
\hline
\end{tabular}

irinotecan sorafenib combination did not lead to additional loss of p27Kip1 (Figure 5B).

\section{Discussion}

Currently, the prognosis for children with AT/RT is very poor. Occasional anecdotal reports of successful treatment are noted; but optimal therapy or even effective therapy has not been achieved in most cases. The chemotherapeutic agents classically used are cyclophosphamide, cisplatin, etoposide, vincristine, carboplatin and ifosfamide [1]. The setback is that tumors seem to be responsive initially but develop resistance [20]. However, recent evidence suggests that improved survival can be achieved with the use of more aggressive treatment approaches, including dose-intense chemotherapy and adjuvant radiation therapy [21-23]. It has also been shown that radiotherapy is crucial to improve the survival rate of children with AT/RT [24,25]. Chi and colleagues have described an innovative treatment approach consisting of an aggressive multimodality approach [26]. This protocol is the first prospective investigation consisting of surgery, radiation therapy combined with multi-agent systemic and IT chemotherapy and has resulted in a significant improvement in time to progression and overall survival of AT/RT patients. In general, the striking potential for long-term consequences of treatments that include radiation in these very young children necessitates trials with new therapeutics and treatment regimens. 


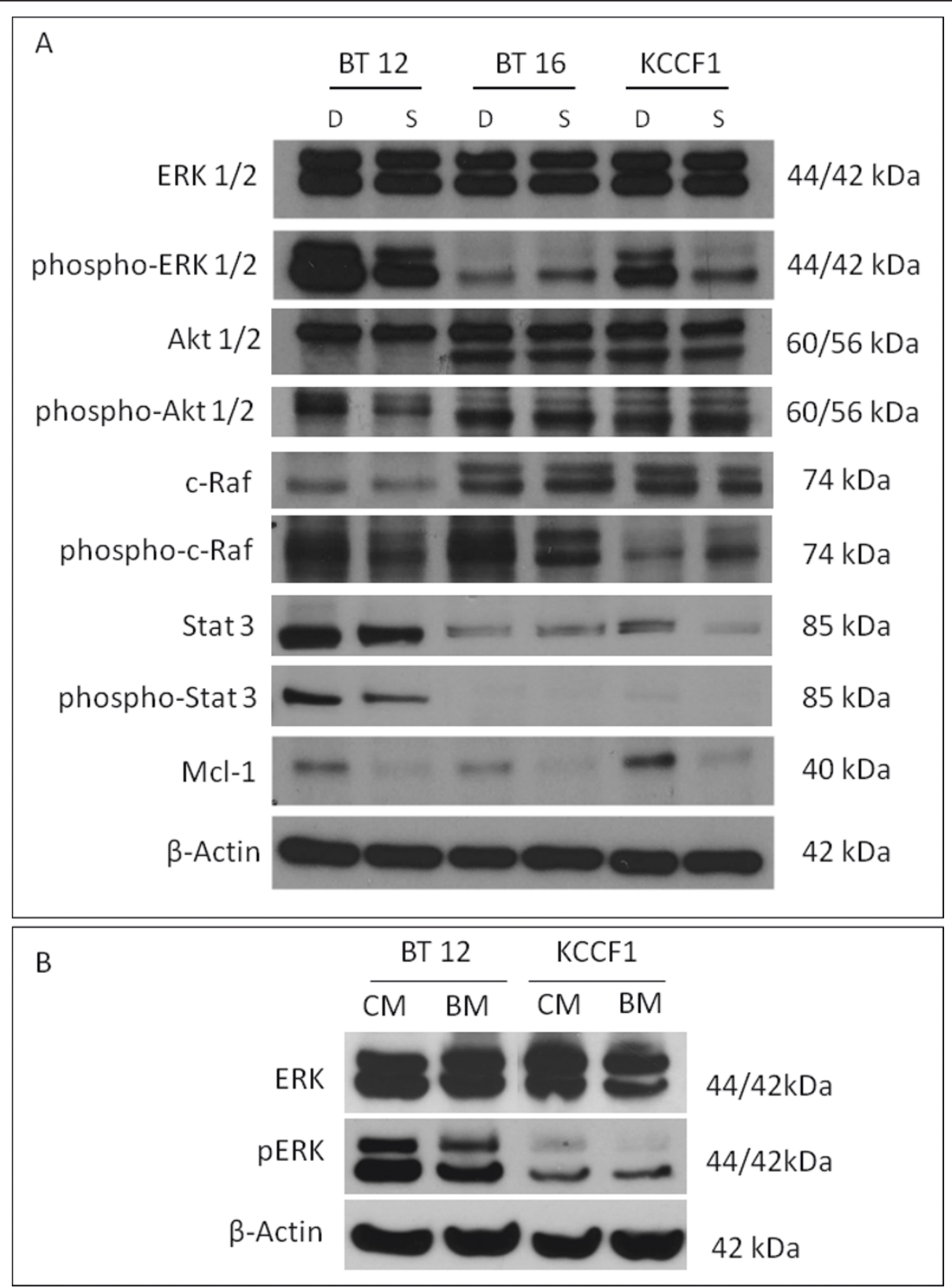

Figure 4 The multi-kinase inhibitor sorafenib induces alterations in cellular signaling and apoptosis regulators in AT/RT cells in response to conditioned medium. Addition of autologous conditioned medium to briefly serum starved cells provides an avenue to evaluate the potential responses that may occur during autocrine/paracrine growth stimulation. In the presence of sorafenib (S), the conditioned medium induced reduced phosphorylation of Erk1/2 (BT12, KCCF1), Akt1/2 (BT12), c-Raf (BT12), Stat3 (BT12, KCCF1) compared to DMSO (D) control. Under these conditions, an increase in phosphorylation of c-Raf was noted in KCCF1 cells. However, the pro-survival protein Mcl-1 was suppressed by sorafenib in all three cell lines (A). Compared to basal medium (BM), conditioned medium (CM) induced phosphorylation of signaling regulators, such as Erk (B). The data shown are typical of three separate experiments.

The role of cytokine receptor mediated growth and survival signals in rhabdoid tumors has been investigated by a number of laboratories. In addition to the effects of IGF-I described previously [27], our studies have shown the expression of significant quantities of VEGF and PDGF by all three cell lines (Table 1). Based on this, we have explored the effects of two multi-kinase inhibitors that have been shown to inhibit growth stimulatory pathways mediated by the receptors of these cytokines. Sorafenib and sunitinib are two oral multitargeted receptor-tyrosine kinase inhibitors that are currently in clinical trials for various malignancies. 


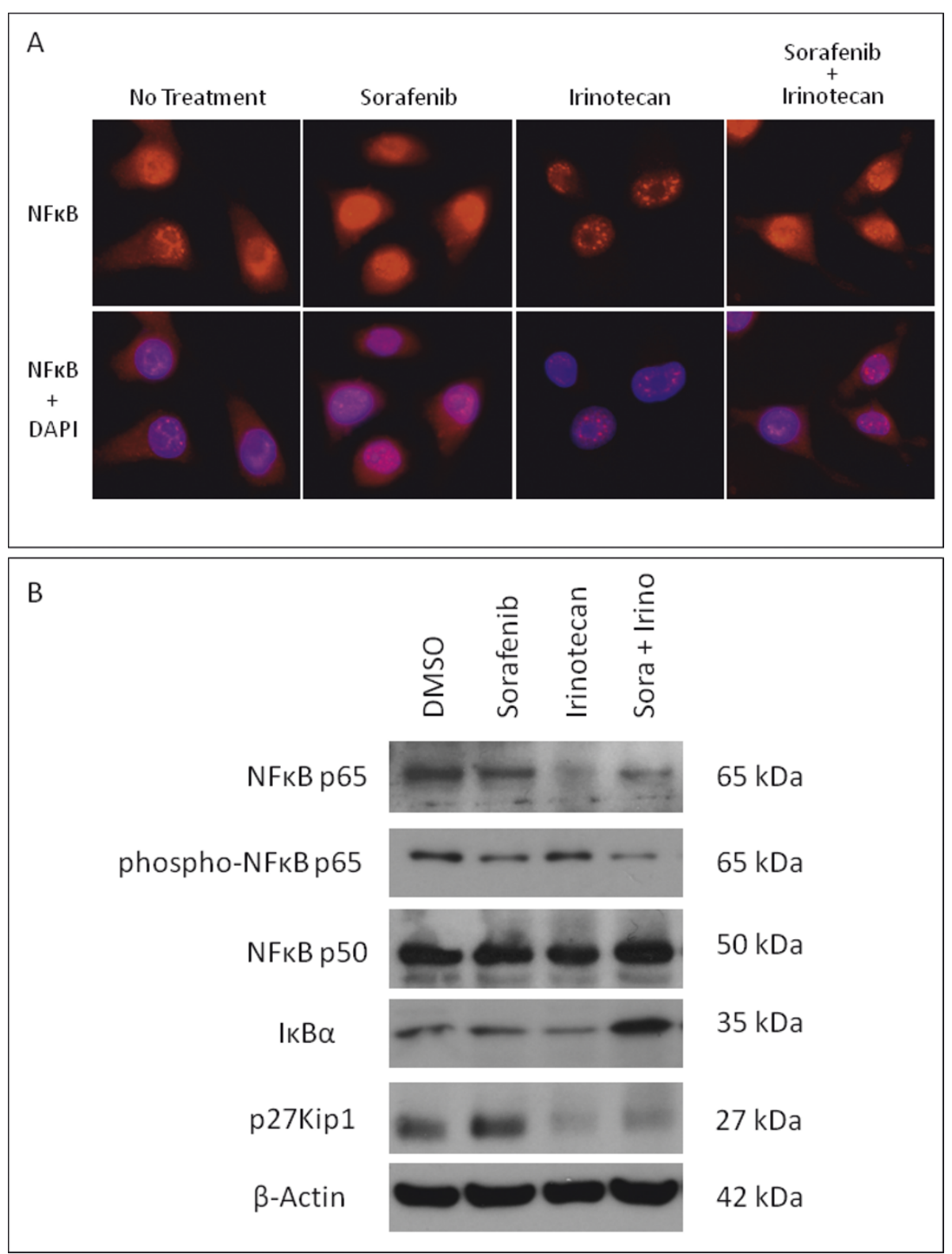

Figure 5 Cytoplasmic NF- $\kappa$ B levels are lowered by irinotecan but sorafenib reduces this effect. BT12 cells were incubated with sorafenib or vehicle for 30 minutes followed by treatment with irinotecan for an additional 2 hours. For indirect immunofluorescence (A), cells were fixed and stained with antibodies to NF- $\kappa$ B followed by fluorescent labeled secondary antibodies. Concurrent DAPI stain was used to localize the nuclei (lower panel). Slides were visualized using a fluorescent microscope and random fields were photographed. The cytoplasmic staining seen in untreated and sorafenib treated cells was significantly reduced following treatment with irinotecan. However, the addition of sorafenib enabled the cells to maintain cytoplasmic staining in the presence of irinotecan. For Western blot analysis (B), cytoplasmic proteins were analyzed using antibodies against NF $\kappa \mathrm{B}$ p65 and p50, phospho-NF $\kappa \mathrm{B}$ p65, $1 \kappa \mathrm{B} \alpha$ and p27Kip1. In the presence of irinotecan, there was a loss of cytoplasmic NF- $\kappa \mathrm{B} p 65$, but in the presence of sorafenib, this loss was greatly reduced, corresponding to a decrease in phosphorylation of NF- $\kappa$ Bp65. In addition, compared to treatment with sorafenib or irinotecan alone, there was increased expression of $\mid \kappa B \alpha$ following treatment with sorafenib and irinotecan. Lastly, following treatment with irinotecan and sorafenib irinotecan combination, there was decreased expression of p27Kip1 compared to sorafenib treatment alone.

Sorafenib is a multi-kinase inhibitor that inhibits the activity of c-Raf, b-Raf, vascular endothelial growth factor receptor family (VEGFR-2 and VEGFR-3), plateletderived growth factor receptor family (PDGFR- $\beta$ ) and stem cell factor receptor (c-Kit). Sunitinib is a multitargeted inhibitor of VEGFR, PDGFR- $\alpha$ and $-\beta, \mathrm{c}-\mathrm{Kit}$ and Flt-3. These two agents offer broad anti-tumor efficacy through their ability to directly and indirectly inhibit 
these targets in concert to ultimately interfere with tumor growth, survival, and angiogenesis [28]. It has been shown in that the antiproliferative effect of sorafenib is mediated through its effect on the MAP kinase pathway (i.e., reduced Erk1/2 phosphorylation) [29]. Our studies have shown a decrease in activated Erk1/2 in two of the three cell lines (Figure 4A). In addition, we have found a decrease in the anti-apoptotic protein Mcl-1 in all three cell lines. Interestingly, the down regulation of Mcl-1 by sorafenib has been shown previously in other tumor models [30]. Mcl-1 has also been implicated in the generation of resistance to chemotherapeutic agents [31]. Though we have shown significant alterations in the activity of key signaling molecules in AT/RT cells, the contribution of off-target effects by sorafenib cannot be ruled out and awaits further analysis in biological correlative studies in xenografts and in future clinical trials of this agent.

Recently, sorafenib has been shown to inhibit proliferation and induce apoptosis in two medulloblastoma cell lines (Daoy and D283) and a primary culture (VC312) of human medulloblastoma at inhibitory concentrations very similar to that we have observed against AT/RT cells $\left(\mathrm{IC}_{50}\right.$ approximately $2.5 \mu \mathrm{M}$ ) [32]. In vivo activity of sorafenib against medulloblastoma cells has also been demonstrated in a mouse xenograft model [32]. Sunitinib has been shown to induce apoptosis and growth arrest in medulloblastoma cells by inhibiting Stat 3 and Akt signaling pathways [33]. In pre-clinical testing studies, Maris and coworkers have observed activity of sunitinib against rhabdoid tumor xenografts [34]. These findings support the potential of sorafenib and sunitinib as effective treatments in AT/RT. However, as a way to increase treatment efficacy and to reduce potential adverse effects of these agents, we explored additional drug combination studies.

Irinotecan has been shown to have the ability to cross the blood-brain barrier and, in preclinical investigations, has demonstrated cytotoxic activity against central nervous system tumor xenografts [35]. Recently, a Phase I trial of irinotecan by Pediatric Oncology Group (POG) was performed in children with refractory solid tumors where stable disease (4-20 cycles) was observed in seven patients with a variety of malignancies, including a patient with CNS AT/RT [36]. In recurrent malignant gliomas, combination therapy with bevacizumab and irinotecan has been shown to prolong progression-free survival in comparison with historical controls $[37,38]$. Our studies have also shown the ability of irinotecan to inhibit the growth of AT/RT cells and significant synergy in drug combinations involving irinotecan with either sorafenib or sunitinib (Table 3). In previous trials, despite the initial response to therapy, most patients treated with irinotecan developed resistance and showed tumor progression [39]. In the colorectal cancer model, treatment with irinotecan has been shown to lead to the activation of NF- $\kappa$ B. [40]. As such, the activation of the NF- $\kappa \mathrm{B}$ pathway constitutes a potential mechanism of inducible resistance by malignant cells exposed to irinotecan [41]. NF- $\kappa \mathrm{B}$ interferes with the effect of most anti-cancer drugs through induction of antiapoptotic genes. Targeting NF- $\kappa \mathrm{B}$ is therefore expected to potentiate conventional treatments in adjuvant strategies. In addition, recent studies have shown that the administration of siRNA directed against the p65 subunit of NF$\kappa \mathrm{B}$ can effectively enhance in vitro and in vivo sensitivity to chemotherapeutic agents [42]. Thus, reducing NF- $\kappa \mathrm{B}-$ mediated activation may help prevent resistance potentially generated upon exposure to irinotecan. This has been confirmed in studies where a pharmacological inhibitor of the IKK2 kinase (AS602868, Serono International SA, Geneva, Switzerland) which blocks NF- $\kappa$ B activation has been found to enhance the action of irinotecan metabolite [43]. We have explored the possibility of decreased $\mathrm{NF}-\kappa \mathrm{B}$ activation as a potential mechanism in the enhanced cytotoxicity of irinotecan in the presence of sorafenib. Our studies have provided evidence for irinotecan mediated loss of cytoplasmic NF- $\kappa$ B in AT/RT cells. However, the presence of sorafenib appears to retain NF- $\kappa$ B in the cytoplasm as shown by Western blot analysis and indirect immunofluoresence studies. Interestingly, in Alzheimer's disease research, a similar observation was noted where the chronic treatment with sorafenib inhibited $\mathrm{c}-\mathrm{Raf}$ and NF- $\kappa \mathrm{B}$ in the brains of the aged APPswe mice [44].

\section{Conclusions}

In this study, we have shown that the AT/RT cell lines produce a number of cytokines and the multi-kinase inhibitors sorafenib and sunitinib induce cell growth inhibition in these cell lines. The effect of sorafenib resulted in the loss of active signaling molecules Erk1/2 in response to conditioned media in two of the three cell lines. We also show that sorafenib inhibits a multitude of signaling molecules in a cell line dependent manner but the loss of the pro-survival protein $\mathrm{Mcl}-1$ was noted in all cell lines studied. We have also shown the synergistic activity of these agents with the topoisomerase I inhibitor irinotecan and provided evidence for the inhibition of NF- $\kappa \mathrm{B}$ activation as one potential advantage in this drug combination. We believe that the data presented here provide the basis for further studies to evaluate the effects of multi-tyrosine kinases in xenograft studies and subsequently for the formulation of clinical studies in patients with AT/RT.

\section{Methods}

\section{Cell lines and cell culture}

BT12 and BT16 cell lines were a gift from Drs. Peter Houghton and Jaclyn Biegel (St. Jude Children's Research Hospital, Memphis, TN, USA). These cell lines have been 
established from infants with CNS AT/RT (BT12 from a six-week-old female; BT16 from a two-year-old male). KCCF1 was established in our laboratory with cells obtained from the Cerebral Spinal Fluid (CSF) of a twomonth-old male infant with AT/RT. Characterization of this cell line has been described previously [9].

Cells were cultured in Opti-MEM medium (Gibco, Invitrogen Corporation, Burlington, Ontario, Canada) supplemented with $5 \%$ fetal bovine serum (Gibco), 100 units $/ \mathrm{ml}$ each of penicillin and streptomycin (Gibco). Confluent cells were trypsinized with $0.25 \%$ Trypsin-EDTA in $\mathrm{Ca} 2+$ and $\mathrm{Mg} 2+$ free balanced salt solution (Gibco) every three to five days. All cell cultures were maintained at $37{ }^{\circ} \mathrm{C}$ in a humidified atmosphere with $5 \% \mathrm{CO}_{2}$.

\section{Multiplex cytokine assay}

Cells $\left(2 \times 10^{6}\right)$ in $2 \mathrm{ml}$ of medium were grown for 48 hours and culture supernatants were analyzed for cytokine levels by multiplex technology as described previously [45]. Briefly, culture supernatants were diluted 1:4 in sample diluent buffer and mixed with beads containing capture antibodies. After incubation and washing steps, beads were mixed and incubated with biotin conjugated detection antibodies. Following the detection antibody incubation, each well was filter-washed and incubated in the dark with streptavidin-phycoerythrin (PE) conjugate. The plates were washed and the contents of each well were resuspended in assay buffer. The plates were read in a Luminex $100^{\mathrm{TM}}$ multiplex assay detection system (Luminex Corp., Austin, Texas, USA) and quantitatively analyzed using STarStation 2.0 software (Applied Cytometry, Sheffield, United Kingdom). In this study, a panel containing the following 65 human cytokines and chemokines was used: EGF, Eotaxin, FGF(basic), Flt-3, Ligand, Fractalkine, G-CSF, GM-CSF, GRO, IFN alpha2, IFN gamma, IL-1alpha, IL-1beta, IL-1Ra, IL-2, IL-3, IL-4, IL-5, IL-6, IL-7, IL8, IL-9, IL-10, IL-12(p40), IL-12(p70), IL-13, IL-15, IL-17, IP-10, MCP-1, MCP-3, MDC, MIP-1alpha, MIP-1beta, PDGF-AA, PDGF-AB/BB, RANTES, CD40L, SDF-1, sIL2R alpha, TGF alpha, TNFbeta, MCP-2, MCP-4, ENA-78, BCA-1, I-309, IL-16, MIP-1delta, TARC, 6Ckine, Eotaxin2, Eotaxin-3, CTACK, IL-23, LIF, TPO, TRAIL, SCF, TSLIP, VEGF, IL-20, IL-21, IL-28A and IL-33.

\section{Antineoplasic agents}

Sorafenib, sunitinib, irinotecan and SN-38 were obtained from ChemieTek (Indianapolis, Indiana, USA) and the Oncology pharmacy at the Alberta Children's Hospital. These agents were dissolved in DMSO (Sigma-Aldrich, Oakville, Ontario, Canada) to a final concentration of 10 $\mathrm{mM}$ and stored in aliquots at $-20^{\circ} \mathrm{C}$. At the time of study, agents were then appropriately diluted in culture medium.

\section{Cell growth inhibition assay}

AT/RT cells were detached from the flask by trypsinization and plated in 96 well plates at a concentration of $1 \times$ $10^{3}$ to $5 \times 10^{3}$ cells per well. Increasing concentrations of study agents were added to these wells to a final volume of $200 \mu \mathrm{l}$ per well. Corresponding dilutions of the vehicle DMSO was used as control. After four days in culture, cell survival was quantified by Alamar Blue Assay (Medicorp, Montreal, Quebec, Canada), according to manufacturer's protocol. Briefly, cells were incubated with $2.5 \%$ Alamar blue (which incorporates a proprietary redox indicator that changes color in response to metabolic activity) for 2 to 24 hours, and the absorbency at $570-620 \mathrm{~nm}$ was measured (Opsys MR Plate Reader, Dynex Technologies, Chantilly, Virginia, USA). Percent cell survival was calculated by:\% Survival $=($ Abs 570-620 test well/Abs 570-620 Control) $\times 100$.

From these values, inhibitory concentrations inducing $50 \%$ cell death $\left(\mathrm{IC}_{50}\right)$ compared to DMSO wells were calculated. For drug combination studies irinotecan at $\mathrm{IC}_{25}$ concentration was added to cultures containing increasing concentrations of sorafenib or sunitinib. $\mathrm{IC}_{50}$ values were then calculated for these agents alone or in combination with irinotecan and used to derive Combination Index (CI) values as described previously [16]. A CI of less than 1 indicates synergy between the two agents under the experimental conditions used.

\section{Western Blot Analysis}

In order to determine the expression of cellular targets of sorafenib and sunitinib in AT/RT cell lines, cells were grown to confluence in six well culture plates (Nunc, Rochester, New York, USA) over a 24 hour period. The media was removed and cells were washed with ice cold PBS and lysed in buffer containing $50 \mathrm{mM}$ Tris, $5 \mathrm{mM}$ EDTA, $0.1 \%$ SDS, 1\% Triton X-100, 0.5\% sodium deoxycholate with phosphatase and protease inhibitors (Sigma). Protein content of the lysates was measured by BCA Protein Assay Kit (Pierce, Rockford, Illinois, USA). Proteins $(30 \mu \mathrm{g} /$ sample) were separated on an $8 \%$ polyacrylamide gel electrophoresis and transferred onto nitrocellulose membranes (Bio-Rad, Mississauga, Ontario, Canada). The membranes were blocked for two hours at $4^{\circ} \mathrm{C}$ with $5 \%$ skim milk powder in PBS containing 0.1\% Tween-20 (Sigma). The blots were incubated with primary antibodies to c-Kit (1:1000, Santa Cruz Biotechnology, Santa Cruz, California, USA), PDGFR- $\beta$ (1:1000, Santa Cruz), VEGFR2 (1:1000, Millipore, Billerica, Massachusetts, USA), Flt-3 (1:1000, Santa Cruz), c-Raf (1:1000, Cell Signaling Technology, Danvers, Massachusetts, USA), p-38 $\alpha$ (1:1000, Santa Cruz) and $\beta$-actin (1: 10000, Sigma). After incubation overnight at $4^{\circ} \mathrm{C}$, membranes were washed and probed with appropriate secondary antibodies conjugated 
to horseradish peroxidase (HRPO) (Sigma), followed by a luminal based substrate (Mandel, Guelph, Ontario, Canada) and developed by exposure to x-ray film (Fisher Scientific, Ottawa, Ontario, Canada). For intracellular signaling studies, cells were grown to confluence in six well culture plates and culture supernatant (spent medium) was removed, filtered and stored at $4{ }^{\circ} \mathrm{C}$ and fresh serum free medium containing $10 \mu \mathrm{M}$ sorafenib or vehicle (DMSO) control was added to the cells. After an additional two hours in culture the spent medium was added $(10 \% \mathrm{v} / \mathrm{v})$. Following further $30 \mathrm{~min}$ in the incubator, cells were lysed as described above and analyzed by Western blot using primary antibodies to Erk1/2 (1:1000, Cell Signaling), phospho-Erk1/2 (1:2000, R \& D Systems, Minneapolis, Minnesota, USA), Akt1/2 (1:1000, Santa Cruz), phospho-Akt1/2 (1:1000, Santa Cruz), c-Raf (1:1000, Cell Signaling Technology), phospho-c-Raf (1:1000, Cell Signaling), Stat3 (1:1000, Cell Signaling), phospho-Stat3 (1:1000, Cell Signaling), Mcl-1 (1:1000, Santa Cruz) and $\beta$-actin. For the analysis of cytoplasmic NF- $\kappa \mathrm{B}$, phospho-NF- $\kappa \mathrm{B}$ and $\mathrm{I} \kappa \mathrm{B} \alpha$, cells were grown in culture for 2 days, after which the media was removed and replaced with serum free media. The cells were then treated with sorafenib (10 $\mu \mathrm{M})$ or DMSO control for 30 minutes, then irinotecan (10 $\mu \mathrm{M})$ for an additional 2 hours. Cell lysates were then analyzed by using primary antibodies to NF- $\kappa$ Bp65 (1:1000, Santa Cruz), phospho-NF $\kappa$ Bp65 (1:1000, Cell Signaling), NF- $\kappa$ Bp50 (1:2000, Millipore), I $\kappa \mathrm{B} \alpha$ (1:1000, Cell Signaling) and $\beta$-actin. For analysis of p27Kip1 expression, cells were treated with either sorafenib $(1 \mu \mathrm{M})$ or irinotecan $(1 \mu \mathrm{M})$ or both for 48 hours. Expression of p27Kip1 was determined using anti-p27Kip1 (1:1000, Cell Signaling).

\section{Immunofluorescence analysis of cytoplasmic NF- $\kappa$ B}

BT12 cells were cultured in six well plates $\left(2 \times 10^{6} /\right.$ well $)$ overnight and treated with (1) vehicle alone, (2) sorafenib (10 $\mu \mathrm{M})$, (3) irinotecan $(10 \mu \mathrm{M})$ and (4) sorafenib for 30 minutes followed by treatment with irinotecan for an additional 2 hours. Indirect immunofluoresence studies were carried out as described previously [46]. Briefly, after various treatments, cells were washed with cold PBS, fixed and incubated with antibodies to NF- $\kappa$ B (Santa Cruz) for 1 hour, followed by fluorescent labeled secondary antibodies (30 min). Concurrent DAPI staining was performed to locate nuclei in each slide. Slides were visualized under a fluorescent microscope and random fields were photographed.

\section{Acknowledgements}

These studies were funded in part by the Alberta Children's Hospital Foundation (ACHF), Kids Cancer Care Foundation of Alberta (KCCFA) and the Brain Tumor Foundation of Canada. AJ holds a Canadian Institutes for Health Research (CIHR) Training Program in Genetics, Child Development and Health Graduate Studentship awarded by the Alberta Children's Hospital
Research Institute (ACHRI) and a McCarthy Tetrault Graduate Studentship awarded by ACHF.

\section{Author details}

${ }^{1}$ Laboratory for Pre-clinical and Drug Discovery Studies, Pediatric Oncology Experimental Therapeutics Investigators Consortium (POETIC) and Division of Pediatric Oncology, Alberta Children's Hospital, 2888 Shaganappi Trail NW, Calgary, T3B 6A8, Canada. ${ }^{2}$ Departments of Biochemistry and Molecular Biology and Oncology, University of Calgary, 3330 Hospital Drive NW, Calgary, T2N 4N1, Canada.

\section{Authors' contributions}

AJ planned and performed drug combination studies and Western blot analysis. DB planned and performed cytotoxicity studies. PB and KR facilitated the indirect immunofluorescence analysis and helped to write the manuscript. AN planned and supervised the studies and wrote the manuscript. All authors have read and approved the final manuscript.

\section{Competing interests}

The authors declare that they have no competing interests.

Received: 30 April 2010 Accepted: 29 December 2011

Published: 29 December 2011

\section{References}

1. Bikowska B, Grajkowska W, Jozwiak J: Atypical teratoid/rhabdoid tumor: short clinical description and insight into possible mechanism of the disease. Eur J Neurol 2011, 18:813-818.

2. Biegel JA: Molecular genetics of atypical teratoid/rhabdoid tumor. Neurosurg Focus 2006, 20:E11.

3. Dufour C, Beaugrand A, Le Deley MC, Bourdeaut F, André N, Leblond P, Bertozzi Al, Frappaz D, Rialland X, Fouyssac F, Edan C, Grill J, Quidot M, Varlet P: Clinicopathologic prognostic factors in childhood atypical teratoid and rhabdoid tumor of the central nervous system: $A$ multicenter study. Cancer 2011, Electronically published ahead of print.

4. Bouvier C, De Paula AM, Fernandez C, Quilichini B, Scavarda D, Gentet JC, Figarella-Branger D: Atypical teratoid/rhabdoid tumor: 7-year event-free survival with gross total resection and radiotherapy in a 7-year-old boy. Childs Nerv Syst 2008, 24:143-147.

5. Guidi CJ, Sands AT, Zambrowicz BP, Turner TK, Demers DA, Webster W, Smith TW, Imbalzano AN, Jones SN: Disruption of Ini1 leads to periimplantation lethality and tumorigenesis in mice. Mol Cell Biol 2001, 21:3598-3603.

6. Foster KS, McCrary WJ, Ross JS, Wright CF: Members of the hSWI/SNF chromatin remodeling complex associate with and are phosphorylated by protein kinase B/Akt. Oncogene 2006, 25:4605-4612.

7. Kohashi K, Oda Y, Yamamoto H, Tamiya S, Izumi T, Ohta S, Taguchi T, Suita S, Tsuneyoshi M: Highly aggressive behavior of malignant rhabdoid tumor: a special reference to SMARCB1/INI1 gene alterations using molecular genetic analysis including quantitative real-time PCR. J Cancer Res Clin Oncol 2007, 133:817-824.

8. Albanese P, Belin MF, Delattre O: The tumor suppressor hSNF5/INI1 controls the differentiation potential of malignant rhabdoid cells. Eur J Cancer 2006, 42:2326-2334.

9. Narendran A, Coppes L, Jayanthan A, Coppes M, Teja B, Bernoux D, George D, Strother D: Establishment of atypical-teratoid/rhabdoid tumor (AT/RT) cell cultures from disseminated CSF cells: a model to elucidate biology and potential targeted therapeutics. J Neurooncol 2008, 90:171-180.

10. D'cunja J, Shalaby T, Rivera P, von Büren A, Patti R, Heppner FL, Arcaro A, Rorke-Adams LB, Phillips PC, Grotzer MA: Antisense treatment of IGF-IR induces apoptosis and enhances chemosensitivity in central nervous system atypical teratoid/rhabdoid tumours cells. Eur J Cancer 2007, 43:1581-1589.

11. Arcaro A, Doepfner KT, Boller D, Guerreiro AS, Shalaby T, Jackson SP, Schoenwaelder SM, Delattre O, Grotzer MA, Fischer B: Novel role for insulin as an autocrine growth factor for malignant brain tumour cells. Biochem J 2007, 406:57-66.

12. Petrelli A, Giordano S: From single- to multi-target drugs in cancer therapy: when aspecificity becomes an advantage. Curr Med Chem 2008, 15:422-432. 
13. Papaetis GS, Syrigos KN: Sunitinib: a multitargeted receptor tyrosine kinase inhibitor in the era of molecular cancer therapies. BioDrugs 2009, 23:377-389.

14. Hartmann JT, Haap M, Kopp HG, Lipp HP: Tyrosine Kinase Inhibitors - A Review on Pharmacology, Metabolism and Side Effects. Curr Drug Metab 2009, 10:470-481.

15. Feun L, Savaraj N: Topoisomerase I inhibitors for the treatment of brain tumors. Expert Rev Anticancer Ther 2008, 8:707-716.

16. Chou TC: Drug combination studies and their synergy quantification using the Chou-Talalay method. Cancer Res 2010, 70:440-446.

17. Nakanishi C, Toi M: Nuclear factor-kappaB inhibitors as sensitizers to anticancer drugs. Nat Rev Cancer 2005, 5:297-309.

18. Lagadec P, Griessinger E, Nawrot MP, Fenouille N, Colosetti P, Imbert V, Mari M, Hofman P, Czerucka D, Rousseau D, Berard E, Dreano M, Peyron JF: Pharmacological targeting of NF-kappaB potentiates the effect of the topoisomerase inhibitor CPT-11 on colon cancer cells. Br J Cancer 2008, 98:335-344.

19. Schiappacassi M, Lovat F, Canzonieri V, Belletti B, Berton S, Di Stefano D, Vecchione A, Colombatti A, Baldassarre G: p27Kip1 expression inhibits glioblastoma growth, invasion and tumor-induced neoangiogenesis. Mol Cancer Ther 2008, 7:1164-1175.

20. Strother D: Atypical teratoid rhabdoid tumors of childhood: diagnosis, treatment and challenges. Expert Rev Anticancer Ther 2005, 5:907-915.

21. Tekautz TM, Fuller CE, Blaney S, Fouladi M, Broniscer A, Merchant TE, Krasin M, Dalton J, Hale G, Kun LE, Wallace D, Gilbertson RJ, Gajjar A: Atypical teratoid/rhabdoid tumors (ATRT): improved survival in children 3 years of age and older with radiation therapy and high-dose alkylatorbased chemotherapy. J Clin Oncol 2005, 23:1491-1499.

22. Shih CS, Hale GA, Gronewold L, Tong X, Laningham FH, Gilger EA Srivastava DK, Kun LE, Gajjar A, Fouladi M: High-dose chemotherapy with autologous stem cell rescue for children with recurrent malignant brain tumors. Cancer 2008, 112:1345-1353.

23. Gidwani P, Levy A, Goodrich J, Weidenheim K, Kolb EA: Successful outcome with tandem myeloablative chemotherapy and autologous peripheral blood stem cell transplants in a patient with atypical teratoid/rhabdoid tumor of the central nervous system. $J$ Neurooncol 2008, 88:211-215.

24. Squire SE, Chan MD, Marcus KJ: Atypical teratoid/rhabdoid tumor: the controversy behind radiation therapy. J Neurooncol 2007, 81:97-111.

25. Chen YW, Wong TT, Ho DM, Huang PI, Chang KP, Shiau CY, Yen SH: Impact of radiotherapy for pediatric CNS atypical teratoid/rhabdoid tumor (single institute experience). Int J Radiat Oncol Biol Phys 2006, 64:1038-1043.

26. Chi SN, Zimmerman MA, Yao X, Cohen KJ, Burger P, Biegel JA, RorkeAdams LB, Fisher MJ, Janss A, Mazewski C, Goldman S, Manley PE, Bowers DC, Bendel A, Rubin J, Turner CD, Marcus KJ, Goumnerova L, Ullrich NJ, Kieran MW: Intensive multimodality treatment for children with newly diagnosed CNS atypical teratoid rhabdoid tumor. J Clin Oncol 2009, 27:385-389.

27. Ogino S, Kubo S, Abdul-Karim FW, Cohen ML: Comparative immunohistochemical study of insulin-like growth factor II and insulinlike growth factor receptor type 1 in pediatric brain tumors. Pediatr Dev Pathol 2001, 4:23-31.

28. Gridelli C, Maione P, Del Gaizo F, Colantuoni G, Guerriero C, Ferrara C, Nicolella D, Comunale D, De Vita A, Rossi A: Sorafenib and sunitinib in the treatment of advanced non-small cell lung cancer. Oncologist 2007, 12:191-200.

29. Wilhelm SM, Carter C, Tang L, Wilkie D, McNabola A, Rong H, Chen C, Zhang $X$, Vincent $P$, McHugh M, Cao Y, Shujath J, Gawlak S, Eveleigh D, Rowley B, Liu L, Adnane L, Lynch M, Auclair D, Taylor I, Gedrich R, Voznesensky A, Riedl B, Post LE, Bollag G, Trail PA: BAY 43-9006 exhibits broad spectrum oral antitumor activity and targets the RAF/MEK/ERK pathway and receptor tyrosine kinases involved in tumor progression and angiogenesis. Cancer Res 2004, 64:7099-7109.

30. Yu C, Bruzek LM, Meng XW, Gores GJ, Carter CA, Kaufmann SH, Adjei AA: The role of $\mathrm{Mcl}-1$ downregulation in the proapoptotic activity of the multikinase inhibitor BAY 43-9006. Oncogene 2005, 24:6861-6869.

31. Warr MR, Shore GC: Unique biology of Mcl-1: therapeutic opportunities in cancer. Curr Mol Med 2008, 8:138-147

32. Yang F, Van Meter TE, Buettner R, Hedvat M, Liang W, Kowolik CM, Mepani N, Mirosevich J, Nam S, Chen MY, Tye G, Kirschbaum M, Jove R:
Sorafenib inhibits signal transducer and activator of transcription 3 signaling associated with growth arrest and apoptosis of medulloblastomas. Mol Cancer Ther 2008, 7:3519-3526.

33. Yang $F$, Jove $V$, Xin $H$, Hedvat $M$, Van Meter TE, Yu H: Sunitinib induces apoptosis and growth arrest of medulloblastoma tumor cells by inhibiting STAT3 and AKT signaling pathways. Mol Cancer Res 2010, 8:35-45.

34. Maris JM, Courtright J, Houghton PJ, Morton CL, Kolb EA, Lock R, Tajbakhsh M, Reynolds CP, Keir ST, Wu J, Smith MA: Initial testing (stage 1) of sunitinib by the pediatric preclinical testing program. Pediatr Blood Cancer 2008, 51:42-48.

35. Houghton PJ, Cheshire PJ, Hallman JD, Lutz L, Friedman HS, Danks MK, Houghton JA: Efficacy of topoisomerase I inhibitors, topotecan and irinotecan, administered at low dose levels in protracted schedules to mice bearing xenografts of human tumors. Cancer Chemother Pharmacol 1995, 36:393-403.

36. Blaney S, Berg SL, Pratt C, Weitman S, Sullivan J, Luchtman-Jones L, Bernstein M: A phase I study of irinotecan in pediatric patients: a pediatric oncology group study. Clin Cancer Res 2001, 7:32-37.

37. Norden AD, Drappatz J, Wen PY: Novel anti-angiogenic therapies for malignant gliomas. Lancet Neurol 2008, 7:1152-1160.

38. Vredenburgh JJ, Desjardins A, Reardon DA, Friedman HS: Experience with irinotecan for the treatment of malignant glioma. Neuro Oncol 2009, 11:80-91.

39. Calvo E, Cortes J, Rodriguez J, Fernandez-Hidalgo O, Rebollo J, MartinAlgarra S, Garcia-Foncillas J, Martinez-Monge R, de Irala J, Brugarolos A: Irinotecan, oxaliplatin, and 5-fluorouracil/leucovorin combination chemotherapy in advanced colorectal carcinoma: a Phase II study. Clin Colorectal Cancer 2002, 2:104-110.

40. $X u Y$, Villalona-Calero MA: Irinotecan: mechanisms of tumor resistance and novel strategies for modulating its activity. Ann Oncol 2002, 13:1841-1851.

41. Wang CY, Mayo MW, Korneluk RG, Goeddel DV, Baldwin AS Jr: NF-kappaB antiapoptosis: induction of TRAF1 and TRAF2 and c-IAP1 and C-IAP2 to suppress caspase-8 activation. Science 1998, 281:1680-1683.

42. Guo J, Verma UN, Gaynor RB, Frenkel EP, Becerra CR: Enhanced chemosensitivity to irinotecan by RNA interference-mediated downregulation of the nuclear factor-kappaB p65 subunit. Clin Cancer Res 2004, 10:3333-3341.

43. Lagadec $P$, Griessinger $E$, Nawrot MP, Fenouille $N$, Colosetti $P$, Imbert $V$, Mari M, Hofman P, Czerucka D, Rousseau D, Berard E, Dreano M, Peyron JF: Pharmacological targeting of NF-kappaB potentiates the effect of the topoisomerase inhibitor CPT-11 on colon cancer cells. Br J Cancer 2008, 98:335-344.

44. Echeverria V, Burgess S, Gamble-George J, Zeitlin R, Lin X, Cao C, Arendash GW: Sorafenib inhibits nuclear factor kappa B, decreases inducible nitric oxide synthase and cyclooxygenase-2 expression, and restores working memory in APPswe mice. Neuroscience 2009, 162:1220-1231.

45. Levina V, Su Y, Nolen B, Liu X, Gordin Y, Lee M, Lokshin A, Gorelik E: Chemotherapeutic drugs and human tumor cells cytokine network. Int J Cancer 2008, 123:2031-2040.

46. Han X, Feng X, Rattner JB, Smith H, Bose P, Suzuki K, Soliman MA, Scott MS, Burke BE, Riabowol K: Tethering of the ING1 protein to Lamin A is required for ING1 function in apoptosis. Nat Cell Biol 2008, 10:1333-1340.

doi:10.1186/1475-2867-11-44

Cite this article as: Jayanthan et al:: Multi-tyrosine kinase inhibitors in preclinical studies for pediatric CNS AT/RT: Evidence for synergy with Topoisomerase-I inhibition. Cancer Cell International 2011 11:44. 